\title{
A Non-Iterative Transformation Method for an Extended Blasius Problem
}

\author{
Riccardo Fazio ${ }^{1}$ \\ ${ }^{1}$ University of Messina
}

April 28, 2020

\begin{abstract}
In this paper, we define a non-iterative transformation method for an Extended Blasius Problem. The original non-iterative transformation method, which is based on scaling invariance properties, was defined for the classical Blasius problem by $\mathrm{T} \backslash$ "opfer in 1912. This method allows us to solve numerically a boundary value problem by solving a related initial value problem and then rescaling the obtained numerical solution. In recent years, we have seen applications of the non-iterative transformation method to several problems of interest. The obtained numerical results are improved by both a mesh refinement strategy and Richardson's extrapolation technique. In this way, we can be confident that the computed six decimal places are correct.
\end{abstract}

\section{Hosted file}

ExBlasius2020.pdf available at https://authorea.com/users/306625/articles/437596-a-non-iterativetransformation-method-for-an-extended-blasius-problem 
figures/ExBlasius/ExBlasius-eps-converted-to.pdf 